\title{
New Organizing: Ein Gespräch über New Work und Agilität
}

\author{
Thomas Bachmann
}

Online publiziert: 4. Januar 2022

(C) Der/die Autor(en) 2022

Zusammenfassung Im folgenden Artikel werden die Entstehung, Entwicklungen und Auswirkungen von New Work und die damit verbunden Konzepte im Dialog zwischen Experten und Expertinnen aus Organisationspsychologie und Beratung diskutiert. Schwerpunkte des Gesprächs sind die derzeitigen Umbrüche in der Arbeitswelt, das Spannungsfeld zwischen New Work und Old Work, Auswirkungen auf Berufswege, Entgrenzung der Arbeit, das agile Mindset, die Rolle des Managements bei agilen Transformationsprojekten sowie die neuen Anforderungen an Beratung in diesem Feld.

Schlüsselwörter Agilität · New Work · New Organizing · Entgrenzung der Arbeit

\section{New organizing: a dialogue about new work and agility}

\begin{abstract}
In the following article emergence, development and impacts of New Work and its associated concepts are discussed in a dialog between experts from organizational psychology and business consulting. The discussion focuses on the current radical changes in work and organizations, the tension between New Work and Old Work, the impact on career paths, the agile mindset the dissolution of work boundaries, the role of management in agile transformation projects, and the new demands on consulting in this field.
\end{abstract}

Keywords Agility $\cdot$ New Work · New Organizing $\cdot$ Dissolution of work boundaries

\footnotetext{
Moderation: Thomas Bachmann

PD Dr. Thomas Bachmann $(\bowtie)$

Institut für Psychologie, Humboldt-Universität zu Berlin, Unter den Linden 6, 10099 Berlin,

Deutschland

E-Mail: thomas.bachmann@hu-berlin.de
} 
Bachmann: New Work und die damit verbundenen Konzepte von New Organizing und Agilität sind in aller Munde. Viele Organisationen hoffen dadurch innovativer, flexibler und attraktiver zu werden. Ist das gerade ein fundamentaler Umbruch in der Arbeitswelt, der sich da vollzieht, oder doch nur ein Hype, der schnell wieder verschwindet, weil er die daran geknüpften Hoffnungen nicht erfüllen kann?

Giernalczyk: New Work ist ein Umbruch, der sich aktuell vollzieht - der aber auch sehr ungleichzeitig ist. Wir sehen große Unterschiede in den Entwicklungsständen, in Tempo und Motivation. Insgesamt besteht in Organisationen aber ein Bewusstsein für die Vorteile von New Work, und dieser Umbruch wird oft auch bewusst angestrebt und gesteuert. Das Führungsprinzip New Work ist noch nicht überall etabliert; eine neue Qualität für Arbeit ergibt sich aber erst, wenn die entsprechenden Methoden sauber kombiniert und konsequent umgesetzt werden.

Bäcker: Bereits vor Corona war ein deutlicher Umbruch der Arbeitswelt zu beobachten. New Work oder New Organizing, Agilität - das bezeichnet lediglich einen Teil der Veränderung. Bei der Betrachtung des Gesamtbildes kommen auch neu etablierte Arbeitsverhältnisse hinzu, z. B. die prekären Anstellungen von Menschen in angeblicher Selbstständigkeit, die fast gänzlich sozial ungesichert sind. Die öffentliche Wahrnehmung wirkt auf mich, als wenn wirklich jeder Beschäftigte von New Work betroffen wäre. Das sehe ich nicht so. Es gibt einige Gruppen und Milieus, die ganz andere Themen haben. New Work ist meiner Meinung nach so im Fokus, weil hier die Beschäftigungsverhältnisse betroffen sind, die nach Andreas Reckwitz kulturdominant sind.

Bachmann: Entsteht hier eine New-Work-Elite, die abgehoben ihre Selbstverwirklichung zelebriert?

Möller: Das geht mir zu weit. Viele ,high potentials“ fühlen sich in den gewachsenen Strukturen vieler Unternehmen nicht länger wohl. Unsere großen Konzerne mit ihren traditionellen Strukturen können kaum Schritt halten mit dem Wertewandel, der sich in den jungen und begabten Menschen vollzieht - das Tempo dabei ist sehr hoch. Da wird eben nicht länger auf Auto, Geld und Status geschaut, sondern auf ganz andere Themen, z. B. Selbstverwirklichung, gute Teamatmosphäre und Anerkennung. Gute Antworten auf die Transformationsnotwendigkeiten zu bieten und wirksame Anreize für Mitarbeitende zu schaffen, fällt einigen Organisationen noch schwer. New Organizing kann da eine Antwort sein, denn das ist die analoge Entwicklung der Organisationen zur gesellschaftlichen Veränderung. Viele scheinen Agilität aber noch nicht in Gänze begriffen zu haben, ich sehe noch Suchbewegungen und oft den Versuch, eine Co-Existenz zum alten System herzustellen. Das funktioniert oft nicht, die halbherzige Umsetzung oder die Co-Existenz von NewWork und Old-Work birgt viel Spannung.

Bachmann: Wenn wir nun den Blick von der Organisations-Ebene auf die Perspektive der beteiligten Personen lenken: Welche sind die zugrundeliegenden Bedürfnisse, die eigentlich adressiert werden müssen? 
Rappe-Giesecke: Die Perspektive auf Organisationen ist inzwischen viel umfassender. Zentral ist die Frage nach Sinn. Statt „Karriere machen“ sehen wir in der Karriereberatung die Sinnfindung und den Wunsch nach Sinnstiftung als großes Thema. Die Menschen suchen nicht mehr ,nur“ die Leiter nach oben, sie wollen sich mit ihrer Mission beschäftigen. Das sind meiner Erfahrung nach nicht nur junge Leute, sondern durchaus auch erfahrene Berufstätige jenseits der 40. Ich wünsche mir eine Akzeptanz der Vielfalt von Organisationsformen. Denn wir haben so vielfältige Aufgaben und Funktionen in unserer Gesellschaft - da sollte Pluralität bestehen. „Form follows function!“

Bachmann: Mit New Organizing in Organisationen wird viel Hoffnung verbunden: Flexibler und innovativer will man werden und so natürlich auch die Wirtschaftlichkeit steigern. Gibt es empirische Erkenntnisse? Sind diese Hoffnungen berechtigt?

Bäcker: Grundsätzlich gibt es zwar viel Literatur zu Agilität und New Work aber empirische Untersuchungen sind kaum vorhanden. Spotify hat ein agiles Organisationsmodell implementiert und konnte, soweit ich weiß, den Umsatz in dieser Zeit steigern. Allerdings ist ein break even wohl noch nicht erreicht. Belegt sind teilweise signifikant gesteigerte Mitarbeiterzufriedenheiten in Unternehmen, die mit New Work-Methoden arbeiten. Mitunter ist gleichzeitig die Kundenzufriedenheit gesunken. Das finde ich bemerkenswert, denn das agile Manifest wurde ja mit Blick auf die Kundenperspektive entwickelt, da steht die User-Experience im Fokus! Ich denke, der Hintergrund ist, dass die Unternehmen in der Umsetzung eines agilen Modells ja zunächst sehr mit sich selbst beschäftigt sind.

Möller: Bei der Suche nach Kausalitäten finde ich Zurückhaltung wichtig. Zum einen sind unternehmerische Selbstdarstellung tendenziell positiv; zum anderen haben wir im wirtschaftlichen Kontext viele Einflüsse und Wechselwirkungen, zum Beispiel den Konjunkturzyklus. Eine eindeutige Zuordnung von Ursache und Wirkung ist da schwierig!

Giernalczyk: Ich finde, Agilität ist bereits an sich eine attraktive Eigenschaft von Organisationen. Die immanente Dynamik bietet bereits Chancen auf Vorteile für Beschäftigte.

Bachmann: New Work ist nicht nur ein Tool-Kit, da sind wir uns einig. Es muss eine entsprechende Organisationskultur entwickelt werden, beteiligte Personen müssen ihr Mindset ändern. Was ist die größte Herausforderung für ein erfolgreiches New Organizing?

Giernalczyk: Agiles Arbeiten, New Work - das hat für mich auch etwas von einem religiösen Versprechen. Schaut her, kommt heran! Hier wird eure Arbeit mit Sinn aufgeladen! Vieles spricht für diese Beschleunigung, für diese Aufladung. Aber in der dann notwendigen Umsetzung liegt die größte Herausforderung, denn da kommen die Aspekte des gesamten Change-Management-Komplexes hinzu! Erst 
große Ziele formulieren und dann in der Umsetzung nicht liefern - das ist ein omnipräsentes Risiko.

Rappe-Giesecke: Nach Schermuly wissen wir, dass kleine Teams und Projekte am funktionalsten sind. Größe ist der maßgebliche Faktor und natürlich Tools und das Mindset. In gewachsenen Organisationen besteht erst einmal eine kritische Masse, die Veränderungen zunächst hemmt. Da begegnen sich bei der Konfrontation mit New Work zwei Logiken: etablierte hierarchische Struktur und Bottom upVeränderung. Wir wissen auch, dass Teams im Veränderungsprozess zur Isolation neigen, und Organisationen haben ebenfalls diese Tendenz, New Work auf Organisationsebene als nicht machbar abzutun. Auch wird es immer Einzelne geben, die beispielsweise das Streben nach Autonomie nicht mittragen, denen New Work nicht entspricht, die da erstmal nicht offen sind. Dem muss bewusst begegnet werden. Dann kann sowohl auf Organisationsebene wie auch auf Ebene des Menschen eine neue Kultur entstehen, ein agiles Mindset geschaffen werden, das Beschäftigte ganz neu sozialisiert.

\section{Bachmann: Was ist denn eigentlich ein agiles Mindset?}

Möller: Im agilen Mindset wird die traditionelle Positionsmacht abgegeben und in die Fähigkeiten eines Teams vertraut. Das ist vor allem eine Herausforderung für Personen, die Jahrzehnte so eine Machtposition innehatten und darüber ihre Identität definieren. Die Zusammenarbeit wird im agilen Mindset kompakter und sichtbarer. Da kann sich keiner verstecken oder auf seiner Position ausruhen. Wenn das agile Mindset etabliert ist, kann es aber gerade für Führungskräfte ein enormer Gewinn sein, sich von der traditionellen Rolle zu lösen. Dann kommt ein menschlicher Kontakt im Team zustande, der enorm bereichernd sein und neue Energie freisetzen kann. Ich glaube, die eigene Gesundheit kann da sehr profitieren - aber wird auch ein neuer Weg in Richtung Selbstausbeutung mit Werkzeugen der New Work bereitet? $\mathrm{Ob}$ in Organisationen in agiler Form auf lange Sicht die Zufriedenheit bestehen bleibt, wird sich zeigen müssen.

Bachmann: Eine wichtige Vokabel bei Laloux (2015) ist die Ganzheitlichkeit. In traditionellen Organisationen erfolgt, systemisch betrachtet, ein Beitritt als Person, die dann einige Facetten ihrer selbst zur Verfügung stellt, verbunden mit einer Trennung von privater Welt und Organisationswelt. Im New Work-Kontext wird die ganze Person gewünscht. Besteht da nicht eine Gefahr der Selbstausbeutung und Entgrenzung?

Möller: Einige Menschen integrieren, viele Menschen separieren! Ein großer Teil der Beschäftigten wird eine Entgrenzung ablehnen - aber es wird sicher Personen geben, die Ganzheitlichkeit begrüßen und wie selbstverständlich leben. Herausfordernd wird es, wenn eine Organisation an der Stelle keine Diversität darstellen kann.

Bäcker: Der Druck steigt - von innen wie von außen. Ich denke ebenfalls: Der größte Teil der Menschen lehnt Entgrenzung ab. Für mich ist die Kernkompetenz 
von Führung in agilen Organisationen deshalb das Balancieren. Das Maß an Tempo, Effizienz und Freiraum auf der einen Seite muss passen zum Bedürfnis nach Halt und Sicherheit auf der anderen Seite. Und dieser Balance-Akt muss mit der Einführung von New Work als Thema mitgedacht werden. Meiner Erfahrung nach werden teils unrealistische Versprechen gemacht - sicher auch im guten Glauben -, die dann ein Strohfeuer an Energie freisetzen. Nach einiger Zeit folgt ein Realitätsabgleich, und die Motivation fällt rapide ab. Das kann z. B. daran liegen, dass in agilen Organisationen oft nur Führungskräfte des unteren und mittleren Managements ihre Macht tatsächlich abgeben, während im Top-Management die Hierarchie weiterhin besteht und gelebt wird. Hier ist aber Konsequenz nötig, das TOP-Management sollte sich nicht von der Veränderung ausnehmen und von Oben auf eine agile Kultur blicken.

Giernalczyk: Wenn wir agiles Arbeiten kritisch würdigen, sehe ich enorme Vorteile - z. B. die damit verbundene geringere Angst vor Fehlern. Die iterative Schleife des Arbeitens hat enorme Wirkmacht. Uns begegnet nun auch etwas, das wir bereits seit der Graswurzel-Bewegung kennen - es ist nun salonfähig, über eigene Fehler zu sprechen. Fehlerkultur wird regelrecht zelebriert. Bis hin zum Meeting „Mein schönster Fehler in diesem Monat.“ Dafür muss das agile Mindset aber organisationsweit bestehen. Wenn noch eine autoritäre Hierarchie-Ebene über allen Teams thront, wird sich niemand aus der Deckung wagen. Anspruch und Umsetzung können weit auseinander liegen. Marcus Zimmermann hat in „Umbruch in der Chefetage“" auf den notwendigen Wechsel im Führungsverständnis hingewiesen (Zimmermann 2016). Es reicht nicht, wenn die Geschäftsführung nach einer Krise - etwa der Kündigung vieler Mitarbeitenden nach einem Umsatzeinbruch - dem mittleren Management ein agiles Verständnis verordnet, ohne sich selbst dahin zu verändern. Dann wird agiles Arbeiten als Anspruch unglaubwürdig.

Rappe-Giesecke: Ich persönlich möchte in einer Organisation nicht ganzheitlich integriert sein. In einer Organisation habe ich eine spezifische Funktion, und an meine Rolle gibt es eine Reihe an definierten Erwartungen durch andere und mich selbst. Wenn Person und Rolle untrennbar miteinander verbunden sind, folgt oft eine Überforderung. Nach meiner Wahrnehmung wird unterschätzt, welchen Schutz eine Rolle und eine Funktion bieten kann. Organisationen hervorgebracht zu haben, in denen Rollen unabhängig von bestimmten Personen sind, ist eine enorme gesellschaftliche Leistung.

Bachmann: Wenn Strukturen und Hierarchien abgebaut werden, ist die Rolle das einzige Konzept, das Orientierung gibt. Wo besteht zu New Work und Agilität außerdem noch Klärungsbedarf in unserer Gesellschaft?

Rappe-Giesecke: Bei den neuen Werkzeugen im agilen Arbeiten gibt es noch keine klare Indikation - und vor allem keine definierte Kontra-Indikation. Das gilt auch allgemein für agiles Arbeiten. Die konzeptionelle Entwicklung erfolgte in Kontexten, in denen das Konzept passend war und funktionierte. Aber wenn wir agiles Arbeiten nun in die Welt hineintragen, müssen wir fragen: Wo sind die Grenzen und wann ist agiles Arbeiten eben nicht angezeigt? 
Giernalczyk: Die Methoden der Beratung sollten intensiv betrachtet werden. Was trennt in der Arbeit des Agile-Coachs nun genau die Retrospektive vom Review? Haben wir lediglich einen modernen Begriff für das, was früher mal als Team-Supervision bezeichnet wurde? Diese Begrifflichkeiten finde ich sehr spannend, denn dahinter stecken Konzepte, die früher nur in der psychosozialen Arbeit gebraucht wurden. Nun ist das hoffähig, und auch in industriellen Profit-Organisationen wird von Selbstreflexion gesprochen. Bei einem mir bekannten digitalen Getränkelieferservice z. B. wird monatlich eine Retrospektive vollzogen - pro Team! Was für ein enormer kultureller Wandel.

Möller: Diese Integration von Perspektiven ist es, wofür wir Beraterinnen und Berater seit Jahrzehnten arbeiten. Ich wünsche mir da weitere Öffnung und mehr Kontaktpunkte!

Bachmann: Wie können wir die traditionelle Arbeit mit Gruppen und Teams zusammenbringen mit moderneren, agilen Kulturelementen von Lernen und Fehleranalyse?

Rappe-Giesecke: Groth beschreibt in seinem kürzlich veröffentlichten Buch „New Organizing“", wie nun das erste Mal eine Veränderungskultur in Organisationen besteht, die von den Mitarbeitenden ausgeht und nicht durch externe Beratung getragen wird (Groth et al. 2021). Wie können wir als externe Berater da unterstützen? Das ist meine zentrale Frage.

Bäcker: Für mich ist zentral, wie wir eine verantwortliche Beratung von Unternehmen umsetzen können, nicht nur von Teams, sondern von ganzen Unternehmen, die sich in Richtung agiler Ziele modernisieren wollen.

Bachmann: Mit der Beratung hin zur selbstständigen Agilität - haben wir hier ein Beratungs-Paradox? Ich denke an ein Unternehmen, das stolz war, agile Konzepte ohne Beratung eingeführt zu haben. Beratung muss sich also neu aufstellen. Stichwort: Kontextgestaltung, weniger im Vorgeben oder Vormachen, Üben, Trainieren, mehr Kontextgestaltung, sodass das Selbstorganisierte eine eigene Form finden kann.

Giernalczyk: Dazu passt, dass agiles Arbeiten ja ursprünglich nicht aus den großen Beratungshäusern kam, sondern von Menschen entwickelt wurde, die nah am Kunden sind. Da sehe ich eine große Herausforderung - Vorstände und Berater werden sich schnell einig, in ihrem Blick von oben. Aber sie sind weit weg vom eigentlichen Geschäft. Für eine gelingende Umsetzung braucht es aber den Kontakt zu den Teams und den Kunden!

Bachmann: Wie werden wir in fünf Jahren über New Work sprechen?

Möller: Wir erleben gerade eine große gesellschaftliche Transformation, die sich mit enormem Tempo vollzieht. Stichwort Vereinbarkeit von Ökonomie und Ökolo- 
gie. Ist Agilität da das Zaubermittel? Ich bin unsicher, ob Organisationsprinzipien Taktgeber sein werden.

Bäcker: In fünf Jahren werden die Themen von New Work noch aktuell sein. Ich stelle mir vor, dass einige Begriffe im engeren Sinne Vergangenheit sind - wir haben dann andere Überschriften. Aber Selbstbestimmung und Freiheit in Organisationen, das wird uns weiter - hoffentlich - begleiten. Auch die Suche nach Stabilität in der Gesellschaft wird bleiben, mehr noch: Sicherheit geben zu können, wird an Bedeutung gewinnen.

Rappe-Giesecke: Die Balance zwischen Stabilität und Wandel wird an Relevanz gewinnen. Wir werden weitere Verfahren und Methoden haben, die Grenzziehung und Stabilisierung zu thematisieren und weiter die Integration von Stabilität und Wandel versuchen.

Giernalczyk: In fünf Jahren werden wir andere Begrifflichkeiten nutzen. Aber der Umgang mit Komplexität in der Arbeitswelt wird weiter ein Thema sein. Diese Komplexität wird ebenfalls weiter zunehmen, und es wird noch viel Bewegung geben zwischen der Agilität und der Suche nach Individualisierung sowie dem Gegengewicht der Standardisierung und der Hierarchie. Um es mit Hegel zu sagen: Agilität war mal Antithese. Das wird in fünf Jahren überholt sein, sie wird mehr und mehr zur These. Daraus werden sich dann neue Antithesen ergeben.

Bachmann: Ich danke Euch allen für das anregende Gespräch.

Der Gesprächsmitschnitt wurde von Sven Volkens transkribiert.

\section{Die Gesprächsteilnehmer}

- PD Dr. T. Bachmann, Humboldt-Universität zu Berlin, Institut für Psychologie, Unter den Linden 6, 10099 Berlin; artop GmbH, Christburger Str. 4, 10405 Berlin, E-Mail: bachmann.thomas@hu-berlin.de

- Dipl.-Psych. R. Bäcker, An der Jüch 15, D-51465 Bergisch Gladbach; 26, rue du Four, F-30190 Sainte-Anastasie, E-Mail: rb@executive-consulting.online

- Prof. Dr. Thomas Giernalczyk, M19 - Manufaktur für Organisationsberatung GmbH, Bauerstraße 19, 80796 München, E-Mail: thomas.giernalczyk@m19-organisationsberatung.de

- Prof. Dr. Heidi Möller, Institut für Psychologie, Universität Kassel, Holländische Straße 36-38, D-34127 Kassel, E-Mail: heidi.moeller@uni-kassel.de

- Prof. Dr. Kornelia Rappe-Giesecke, Qualenriehte 25, 31535 Neustadt, E-Mail: oe@rappe-giesecke.de

Funding Open Access funding enabled and organized by Projekt DEAL.

Open Access Dieser Artikel wird unter der Creative Commons Namensnennung 4.0 International Lizenz veröffentlicht, welche die Nutzung, Vervielfältigung, Bearbeitung, Verbreitung und Wiedergabe in 
jeglichem Medium und Format erlaubt, sofern Sie den/die ursprünglichen Autor(en) und die Quelle ordnungsgemäß nennen, einen Link zur Creative Commons Lizenz beifügen und angeben, ob Änderungen vorgenommen wurden.

Die in diesem Artikel enthaltenen Bilder und sonstiges Drittmaterial unterliegen ebenfalls der genannten Creative Commons Lizenz, sofern sich aus der Abbildungslegende nichts anderes ergibt. Sofern das betreffende Material nicht unter der genannten Creative Commons Lizenz steht und die betreffende Handlung nicht nach gesetzlichen Vorschriften erlaubt ist, ist für die oben aufgeführten Weiterverwendungen des Materials die Einwilligung des jeweiligen Rechteinhabers einzuholen.

Weitere Details zur Lizenz entnehmen Sie bitte der Lizenzinformation auf http://creativecommons.org/ licenses/by/4.0/deed.de.

\section{Literatur}

Groth, T., Krejci, G. P., \& Günther, S. (Hrsg.). (2021). New Organizing: Wie Großorganisationen Agilität, Holacracy \& Co. einführen - und was man daraus lernen kann. Heidelberg: Carl-Auer.

Laloux, F. (2015). Reinventing Organizations: Ein Leitfaden zur Gestaltung sinnstiftender Formen der Zusammenarbeit. München: Vahlen.

Zimmermann, M. (2016). Umbruch in der Chefetage. Freiburg: Haufe. 